\title{
Correction to: Every variance function, including Taylor's power law of fluctuation scaling, can be produced by any location-scale family of distributions with positive mean and variance
}

\author{
Joel E. Cohen ${ }^{1,2,3}$ (D)
}

Published online: 20 January 2022

(c) Springer Nature B.V. 2022

\section{Correction to: Theoretical Ecology 13(1):1-5, 2020 https://doi.org/10.1007/s12080-019-00445-7}

Dr. Thierry Huillet of CY Cergy Paris University (personal communication, November 25, 2021) kindly pointed out an error in the last line of Eq. (5) in my attempt to prove the portion of Theorem 1 pertaining to the quadratic generalization of Taylor's law (QTL), Eq. (2), $\log \operatorname{Var}(X(p))=\log a+b_{1} \log E(X(p))+b_{2}(\log E(X(p)))^{2}$. I made the same mistake in the last line of Eq. (8) in my attempt to prove a generalization of Theorem 1 .

Happily, both Theorem 1 and the generalization of Theorem 1 remain true under additional conditions on the coefficients, which were omitted in the original statements. The claims follow immediately from Theorem 3 , the proof of which is valid. Independent of Theorem 3, I give here a direct, elementary proof of Theorem 1 with additional conditions on $a, b_{1}, b_{2}$ and $P$.

For real-valued random variables $X, Y$, define $X \sim Y$ if and only if, for some real $c, d \neq 0, X$ has the same distribution as $c+d Y$.

Theorem 1 Let $Z$ be any real-valued random variable with expectation $E Z:=\mu \in(0, \infty)$ and variance Var $Z:=\sigma^{2} \in(0, \infty)$. Let $a, b_{1}, b_{2}$ be nonnegative real numbers such that $a>0$ and

The original article can be found online at https://doi.org/10.1007/ s12080-019-00445-7.

Joel E. Cohen

cohen@ rockefeller.edu

1 Laboratory of Populations, Rockefeller University and Columbia University, 1230 York Avenue, Box 20, New York, NY 10065, USA

2 Earth Institute and Department of Statistics, Columbia University, New York, NY 10027, USA

3 Department of Statistics, University of Chicago, Chicago, IL 60637, USA at least one of $b_{1}, b_{2}$ is positive. If $b_{2}>0$, then there exists a family of random variables $\left\{X(p) \mid p \in P \subset\left[p_{0}, \infty\right), p_{0}>0\right\}$ such that the QTL holds for the chosen $a, b_{1}, b_{2}$ and $X(p) \sim Z$ for every $p \in P$. If $b_{1}>0, b_{2}=0$, then $T L$, namely, $\log \operatorname{Var}(X(p))=\log a+b_{1} \log E(X(p))$, holds.

Proof Given $a, b_{1}, b_{2}$, pick a positive number $g$ that is large enough to guarantee that $\log a+b_{1} g p+b_{2} g^{2} p^{2}>0$ for every $p \in P$. Such a choice is possible because of our assumptions about $a, b_{1}, b_{2}$ and $P$. Define, for every $p \in P$,

$X(p):=e^{g p}+\sqrt{\log a+b_{1} g p+b_{2} g^{2} p^{2}}(Z-\mu) / \sigma$

Then for each $p \in P, X(p)$ is a linear function of $Z$, so $X(p) \sim Z, E X(p)=e^{g p}, \log E X(p)=g p \neq 0$, and the QTL holds because

$$
\begin{aligned}
\operatorname{Var} X(p) & =\log a+b_{1} g p+b_{2} g^{2} p^{2} \\
& =\log a+b_{1} \log E(X(p))+b_{2}(\log E(X(p)))^{2}
\end{aligned}
$$

A polynomial Taylor's law generalizes Theorem 1. The variance function

$\log \operatorname{Var}(X(p))=\log a+\sum_{i=1}^{\infty} b_{i}[\log E(X(p))]^{i}$

follows similarly by defining

$X(p):=e^{g p}+\sqrt{\log a+\sum_{i=1}^{\infty} b_{i}[g p]^{i}}(Z-\mu) / \sigma$

under conditions on $a, b_{i}$, and $P$ sufficient to guarantee that $\log a+\sum_{i=1}^{\infty} b_{i}[g p]^{i}>0$.

The affiliations in the original publication should be replaced with: Laboratory of Populations, Rockefeller University and Columbia University, 1230 York Avenue, 
Box 20, New York, NY 10065, USA; Earth Institute and Department of Statistics, Columbia University, New York, NY 10027, USA; and Department of Statistics, University of Chicago, Chicago, IL 60637, USA.

Author Contributions This manuscript was written and revised by its sole author.
Funding The author declares that no funds, grants, or other support were received during the preparation of this manuscript.

\section{Declarations}

Competing interest The author has no relevant financial or non-financial interests to disclose. 